\title{
Los impuestos territoriales en Colombia y la inequidad social, ¿la voluntad de la clase dominante erigida en ley?*
}

\author{
Mauricio García Garzón ${ }^{* *}$ \\ Guillermo Fino Serrano
}

Recibido: 5 de agosto de 2014. - Revisado: 20 de agosto de 2014

Aprobado: 7 de septiembre de 2014.

\section{Resumen}

El presente artículo evidencia un análisis crítico de la regresividad del sistema tributario tanto a nivel departamental como a nivel municipal. Para los primeros, el panorama continúa sustentado en la capacidad que tengan los gobiernos regionales de incrementar el vicio para que la población incremente el consumo de bebidas alcohólicas, tabaco y juego de suerte y azar, que constituyen la columna vertebral de sus recursos y que se clasifican como impuestos indirectos, los cuales son por naturaleza regresivos. De otro lado, los ingresos municipales presentan un resultado similar frente a la inequidad tributaria, por cuanto la mayor parte de los ingresos propios, provienen de los impuestos al consumo (industria y comercio), que se caracterizan igualmente por ser de naturaleza regresiva.

"El presente artículo pertenece al proyecto de investigación sobre impuestos y desigualdad social que trabajan los grupos GACE y GESEF de las facultades de Contaduría Pública y Finanzas y Comercio Internacional de la Corporación Universitaria Republicana, Bogotá. El proyecto de investigación mencionado es financiado por la citada Corporación.

“"Economista de la Universidad de América, especialista en Gerencia Pública y Control Fiscal de la Universidad del Rosario, magister en Administración Pública de la Escuela Superior de Administración Pública -ESAP-. Docente investigador del Grupo GACE de la Facultad de Contaduría Pública de la Corporación Universitaria Republicana. Correo electrónico: mgarcia@urepublicana.edu.co

*mo Abogado, Universidad Libre de Colombia, especialista en Derecho Administrativo, Universidad Santo Tomás, magister en Derecho Administrativo Universidad Libre, candidato a doctor Universidad Libre. Docente investigador y líder del Grupo de Investigación en Finanzas Publicas GESEF de la Corporación Universitaria Republicana. Correo electrónico: gfino@urepublicana.edu.co 
Palabras clave: hacienda pública, impuestos territoriales, inequidad tributaria, función redistributiva.

\title{
Property taxes in Colombia and Social inequality, THE WILL OF THE RULING CLASS MADE INTO A LAW?
}

\begin{abstract}
This document is one critical analysis of the regressivity of the tax system performs both work departmental level and at the municipal level. For the former, the outlook remains supported by the ability of regional governments to increase the vice so that the population increase the consumption of alcohol, snuff and game of chance, which are the backbone of its resources and they are classified as indirect taxes, which are regressive nature. On the other hand, municipal income present a similar result against tax inequity, since most of the own revenues come from consumption taxes (industry and trade), which is also characterized by regressive in nature.
\end{abstract}

Keywords: Public finance, property taxes, tax inequity, redistributive function.

\section{OS IMPOSTOS TERRITORIAIS NA COLÔMBIA E A DESIGUALDADE SOCIAL: A VONTADE DA CLASSE DOMINANTE ERGUIDA POR LEI?}

\section{Resumo}

O presente artigo evidencia uma análise crítica da regressividade do sistema tributário tanto na esfera estadual como municipal. Para o primeiro, o panorama continua sustentado na capacidade dos governos regionais de incrementar o vicio para que a população aumente o consumo de bebidas alcoólicas, tabaco e jogos de azar, que constituem a coluna vertebral de seus recursos e que se classificam como impostos indiretos, os quais são por natureza, regressivos. Por outro lado, os ingressos municipais apresentam um resultado similar frente à desigualdade tributária, portanto a maior parte dos ingressos próprios provém dos impostos sobre consumo (indústria e comércio), que se caracterizam igualmente por ser de natureza regressiva.

Palavras-chave: Fazenda pública, impostos territoriais, desigualdade tributária, função redistributiva. 


\section{Introducción}

La hacienda pública se encarga de estudiar la equidad de los impuestos, tanto en lo relativo al reparto de las cargas públicas entre los miembros de la sociedad como de la adecuada y acertada redistribución de los ingresos, aspecto de inusitada importancia en el devenir diario de la sociedad.

El presente artículo evalúa, desde la perspectiva de la función redistributiva de la hacienda pública, cómo la estructura de los impuestos territoriales en Colombia contribuye o no a la obligación de aportar a las cargas públicas territoriales a través de la progresividad de los impuestos, para lo cual se adelanta un análisis de la estructura tributaria de los departamentos y municipios del país.

El estudio se dividió en tres partes, a saber: la primera tiene que ver con los enfoques teóricos y normativos de la función redistributiva de la hacienda pública, la segunda analiza la estructura de los impuestos departamentales y la tercera analiza la estructura de los impuestos municipales.

Como marco teórico se trabajan las diferentes concepciones sobre las máximas de los impuestos en general: igualdad, equidad, certidumbre, comodidad del pago y economía de la recaudación y eficiencia de los clásicos, principalmente de Smith, y la función redistributiva de la hacienda pública de Richard Musgrave, buscando con ello, compilar las bases teóricas que permitan conceptualizar la realidad objeto de estudio.

Este es un estudio analítico, basado en fuentes secundarias, con un enfoque metodológico cuantitativo, en lo que tiene que ver con la recolección y procesamiento de información estadística, para lo cual se adelantó la revisión documental y recolección de cifras, a partir de las ejecuciones presupuestales remitidas por las respectivas entidades territoriales al Departamento Nacional de Planeación-DNP-, que se encarga de consolidar anualmente toda estas cifras a través de los informes de desempeño fiscal de los departamentos y municipios del país. Se toma como referencia el año 2013, que corresponde a la última vigencia que presenta cifras consolidadas de las finanzas públicas territoriales.

El impacto esperado es generar una propuesta académica donde se planteen elementos de discusión en los temas fiscales de los territorios del país, que trasciendan a las autoridades gubernamentales, con el fin de que se diseñen políticas públicas que conlleven a mejorar el bienestar de la población, aprovechando de una u otra manera su estructura tributaria. 


\section{Función redistributiva de la hacienda pública: enfoques teóricos y normativos}

El objeto principal de la hacienda pública es el estudio del sector público como recaudador o inversor de los recursos fiscales, y cuyo propósito fundamental se encuentra sustentado en la función redistributiva, que se da primordialmente por el reparto personal de rentas, que busca disminuir el ingreso global de unos sectores de la población y reasignarlos hacia sectores menos favorecidos. Esto se logra a través de la progresividad de ciertos tributos, en especial los impuestos directos, que a nivel territorial tienen que ver con los recaudos departamentales de registro y anotación y vehículos automotores, y a nivel municipal con el predial.

En este sentido, Restrepo (2008) señala lo siguiente:

En el fondo, las razones que explican los propósitos redistributivos de la hacienda pública no son otros que los de ofrecer, o al menos acercar, la igualdad de oportunidades de que gozan los miembros de la sociedad para participar en el crecimiento económico. Cuando el Estado gasta más que proporcionalmente en salud pública y en educación primaria, por ejemplo, en el fondo lo que está haciendo es restablecer la igualdad de oportunidades entre quienes nacen rodeados de todo tipo de privilegios y quienes carecen de ellos. Puesto que los bienes colectivos que proporciona la hacienda pública entran a asignar una mayor proporción de ellos a un segmento de la población desvalida maximiza el bienestar del conjunto y establece externalidades positivas para la sociedad (p. 32).

Para poder entender un poco más el tema redistributivo de la hacienda pública, debemos referirnos a uno de los autores clásicos como Adam Smith, citado en Silva, Páez y Rodríguez (2008), cuando señala que existen cuatro máximas con respecto a los impuestos en general: igualdad, equidad, certidumbre, comodidad del pago y economía de la recaudación-eficiencia (1776, p. 726).

Musgrave, 1985 y 1987, citados en Silva et al. (2008, p. 31) compilan las apreciaciones de Smith y de otros autores importantes de la siguiente manera:

Aunque las máximas de Smith comienzan con la capacidad de pago, avanzan hacia una regla del beneficio. Sin embargo, para Smith la contribución se debe fijar en 
proporción a los ingresos recibidos, excluye los ingresos salariales de subsistencia. Otra exención la constituye el impuesto sobre los alquileres de vivienda. La mayoría de autores contractualistas (Hobbes) apoyaron el punto de vista proporcional, también lo hizo Bentham, partidario de la exención de los salarios de subsistencia. El punto central de la regla según el beneficio, es definir si el impuesto se determina por el costo del servicio ofrecido a una persona determinada o si es sobre lo que una persona (dadas su renta y preferencias) está dispuesta a pagar. En el último caso, el impuesto según el beneficio se convierte en un precio tipo Lindahl y la imposición progresiva gira entonces sobre las elasticidades precio y renta de la demanda, factores que dependen de los servicios concretos en cuestión, no pudiendo generalizarse sobre el presupuesto como un todo (Musgrave, 1985, p. 20).

El principio del beneficio tiene la ventaja que enlaza los dos lados del presupuesto (impuesto y el gasto) y así los relaciona con la teoría de los bienes públicos. Por su parte, el principio de capacidad de gasto (pago) se concentra en el lado de los ingresos atendiendo a la distribución de la carga tributaria. El precio Lindahl, después de todo, fue un impuesto al beneficio por excelencia. Sin embargo, las finanzas de los precios fijos o de cuotas, relacionados con el nivel de consumo individual de bienes públicos interfieren con su provisión eficiente. El principio del beneficio tampoco admite el uso redistributivo del proceso fiscal (Musgrave, 1987).

El principio de capacidad de pago tiene también una larga historia, Montesquieu y Say fueron partidarios de la progresión, mientras que Bodin defendió una tributación proporcional. Sin embargo la historia moderna de la capacidad de pago empieza con la formulación de Mill. "Escribiendo en los años 1840, Mill fue sensible a una estructura política y filosófica totalmente diferente a la de Adam Smith" (Musgrave, 1985, p. 21).

Richard Musgrave, en los años cincuenta recoge varios de estos aspectos, y es así como la función redistributiva de la hacienda pública encuentra sustento en la teoría de este autor, cuyos preceptos se fundamentan no solo en el suministro de bienes colectivos por parte de la acción estatal, sino en el desarrollo de los propósitos de redistribución, estabilidad y desarrollo económico.

Para Musgrave, el Estado cumple tres funciones, a saber: estabilización macroeconómica, distribución y asignación de recursos. La primera es exclusiva del nivel nacional que cuenta con los instrumentos de política económica para desarrollarla; 
la segunda puede ser desarrollada por la nación y los territorios y la tercera que comprende la provisión de bienes y servicios públicos puede ser atendida por diferentes niveles de gobierno, atendiendo a la naturaleza de dichos bienes y servicios; siendo su principal objetivo la asignación de recursos necesarios para la satisfacción de las necesidades sociales que, en común con los deseos privados, reflejan las preferencias de los individuos (Musgrave, 1959, p. 118).

En este sentido, el gobierno del nivel central debe asegurar un mínimo de provisión de bienes públicos (puros, como la defensa o sociales, como la salud) a toda la nación y lo debe hacer con el fin de cumplir con la tarea de redistribución de la renta y la riqueza de la hacienda pública. Lo anterior, porque los bienes objeto de la provisión, generalmente, son los que mayor beneficio traen a las personas con menos ingresos. Además, los autores sostienen que para cumplir dicho fin las transferencias de carácter condicionadas son las más eficaces (Musgrave y Musgrave, 1992).

De otro lado, los principios tributarios poseen un fundamento constitucional, dado por los artículos 95 y 363 de la Carta Magna; los cuales estipulan que los ciudadanos deben contribuir al financiamiento de los gastos e inversiones del Estado dentro de conceptos de justicia y equidad (artículo 95), y que el sistema tributario se funda en los principios de equidad eficiencia y progresividad (artículo 363).

La justicia tributaria se refiere a la obligación que tiene cada individuo de pagar impuestos para el financiamiento de los gastos e inversiones del Estado, ya que así se beneficia la sociedad en general y con ello el individuo mismo; y a su vez, las leyes tributarias deben buscar que las cargas tributarias sean justas y no excesivas para los ciudadanos, gravando a cada uno según su capacidad de pago. La autoridad tributaria debe exigir el cumplimiento a todos los sujetos obligados a pagar el tributo.

La equidad tributaria se refiere a cómo debe recaer la carga impositiva en el contribuyente teniendo en cuenta su capacidad de pago, "el mismo término equidad lleva a pensar en equilibrio y desde este punto de vista, el equilibrio se da cuando las magnitudes están bien repartidas, en nuestro caso cuando las cargas tributarias estén bien repartidas en los contribuyentes" (Beltrán y Torres, 2004, p. 165).

El mismo autor enuncia que la equidad tributaria se da cuando las cargas tributarias recaen en los contribuyentes en proporción a su capacidad contributiva. Así, entonces:

.... El principio de la equidad tributaria se da cuando las cargas tributarias recaen en los contribuyentes en proporción a su capacidad de pago. Se da la equidad 
horizontal cuando los contribuyentes pagan iguales impuestos siendo iguales sus niveles de renta.

Se da la equidad vertical cuando los contribuyentes pagan distinta cantidad de impuesto, dados diferentes niveles de renta; el Estado, al hacer la imposición tributaria, debe buscar el mayor acercamiento posible a una justicia natural a través de las Leyes (Beltrán, et al., 2004, p. 166).

La Corte Constitucional mediante sentencia C-296 de 1999 se refiere al principio de equidad tributaria así:

Por consiguiente, el estudio de constitucionalidad de una disposición legal desde la perspectiva de la equidad tributaria, si bien debe considerar la generalidad e igualdad en la tributación, entendida esta última como igualdad de trato para contribuyentes colocados en iguales circunstancias, tanto económicas como fácticas y diferenciación de cargas y beneficios tributarios con base en criterios razonables y objetivos, también debe analizar la situación norma-caso, toda vez que ahí el concepto de equidad se aplica inevitablemente (Fino, 2012, p. 47).

El principio de progresividad consiste en que los contribuyentes deben ser gravados con impuestos según su capacidad de pago, de tal manera que a mayor capacidad contributiva le corresponde pagar mayor impuesto. Así entonces, el que tiene más paga más, el que tiene menos paga menos, y el que no tiene nada no paga nada.

Aquellos impuestos que recaen sobre las actividades económicas del intercambio, sin que tenga en cuenta el factor riqueza del contribuyente, posee un efecto contrario a la progresión, es decir, se está frente a impuestos regresivos, los cuales afectan a las clases menos favorecidas.

En términos técnicos, se busca que el conjunto del sistema tributario tenga con relación al PIB una elasticidad superior a uno. Otro parámetro de evaluación de la progresividad desde luego, consiste en que, en especial por lo que hace a la tributación directa, se consulte siempre la capacidad de pago de los obligados tributariamente, o dicho en otras palabras, que quien más tiene más pague. De ahí que se diga que existen vínculos cercanos entre el principio de la equidad y el de la progresividad. Un sistema tributario que hiciera recaer en su conjunto mayores 
cargas relativas sobre quienes tienen menos capacidad de pago sería a la vez un sistema que se alejaría del cumplimiento de la meta tanto de la equidad como de la progresividad (Restrepo, 2008, p. 395).

El principio de eficiencia hace relación a la economía del tiempo y recursos, así como a la simplificación de los procedimientos para alcanzar los objetivos tributarios. De esta manera, el pago de impuestos no les debe generar pérdidas de tiempo a los contribuyentes en el cumplimiento de sus obligaciones tributarias.

El costo mínimo de recaudación y máxima eficiencia en su aplicación significa que la parte del impuesto que se destine para su recaudo sea mínima y que los propósitos para los cuales se establece la tributación se alcancen en forma inmediata, generando con ello confianza en las personas, sujetos de los impuestos (Ramírez, 2011, p. 96).

La consecuencia frente al citado interrogante es que los impuestos quedan mal liquidados, al respecto dice un informe al Congreso de 2010 (citado en Fino, 2012), a nivel territorial prevé el principio de equidad se rompe de diversas maneras. Una de ellas es que a través del impuesto predial que se viene cobrando sobre un incremento catastral anual equivalente a la inflación, sin tener en cuenta el verdadero valor de la tierra, lo que implica que el avalúo no se haya incrementado proporcionalmente en el transcurso de los años, lo que arroja como resultado que los municipios poco o nada reciban de este mayor valor implícito.

Finalmente, es importante mencionar que la tributación tiene objetivos de carácter permanente, tales como: la financiación del gasto público, la redistribución del ingreso, la financiación de ciertos bienes y servicios públicos y de influir sobre los grandes agregados económicos mediante la intervención del consumo y la inversión pública y privada (Ramírez, 2011).

Luego de abordar los aspectos teóricos y normativos, pasamos a mirar cuál es la estructura de los impuestos departamentales y municipales del país, veamos. 


\section{Estructura de los impuestos departamentales}

En este apartado se adelanta un análisis de la estructura tributaria de los departamentos y municipios del país, con miras a contrastar la siguiente hipótesis: "la obligación de aportar a las cargas públicas territoriales, tanto a nivel departamental como municipal son inequitativas”.

Esta hipótesis está articulada con uno de los propósitos fundamentales de la hacienda pública, como es la función redistributiva del ingreso, que se persigue alcanzar a través de la aplicación del principio tributario de progresividad.

A continuación se hace un análisis de la caracterización de los principales impuestos departamentales:

Los impuestos de los departamentos se asocian de alguna manera con el vicio, ya que sus principales recaudos están representados por el consumo de cerveza, licor, cigarrillos y tabaco, los cuales representan el $57 \%$ del total impositivo. Otros impuestos importantes son: registro y anotación, vehículos automotores y sobretasa a la gasolina.

El sustento normativo de los impuesto al consumo de cerveza, licores, vinos, aperitivos y similares, cigarrillos y tabaco, así como el de registro y anotación se encuentran definidos en la Ley 223 de 1995, por medio de la cual se expiden normas sobre racionalización tributaria y se dictan otras disposiciones sobre la materia. Así entonces, el impuesto al consumo de cerveza se reglamenta en los artículos 185 al 201, el impuesto al consumo de licores, vinos, aperitivos y similares a través de los artículos 202 al 206, el impuesto al consumo de cigarrillos y tabaco desde el artículo 207 al 2012 y el impuesto de registro y anotación a partir del artículo 226 al 236.

En dicha Ley se estipula la propiedad del impuesto, el hecho generador, los sujetos activo y pasivo, la acusación, la base gravable, las tarifas, el periodo, la declaración y el pago del impuesto. Adicionalmente en la Ley 1111 de 2006, se establece que los sujetos activos deberán establecer la obligación a los sujetos pasivos del uso de tecnologías de señalización para el control, que permitan garantizar el pago de los impuestos.

Una vez definido el sustento normativo de los principales impuestos departamentales, se establece su estructura tomando como referencia el año 2013, que corresponde a la última vigencia que presenta cifras consolidadas de las finanzas públicas territoriales. 
Para la vigencia de 2013, el recaudo de los ingresos tributarios de todos los departamentos del país llegó a la suma de \$5,7 billones (DNP, 2013), con el siguiente peso porcentual:

Figura 1. Participación porcentual impuestos departamentales - 2013

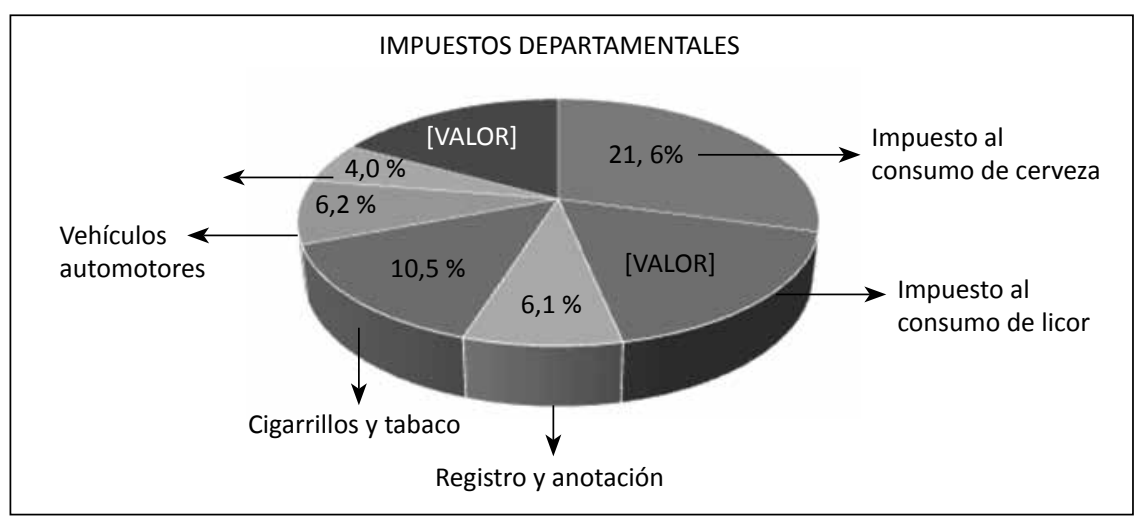

Fuente: desempeño fiscal 2013, DNP

De los anteriores impuestos departamentales se clasifican como directos: el impuesto de registro y anotación y el de vehículos automotores que representan el $12,3 \%$ del total; los demás impuestos clasificados como indirectos representan el restante $87,7 \%$, lo que indica que la estructura tributaria departamental es altamente regresiva, favoreciendo la inequidad en la redistribución de la riqueza en nuestro país.

A lo anterior, se le debe agregar que la estructura tributaria departamental presenta un profundo contrasentido, mientras que los gobiernos regionales buscan incrementar la capacidad para que la población incremente el consumo de bebidas alcohólicas, tabaco y juego de suerte y azar, que constituyen la columna vertebral de sus recursos, los cuales se clasifican como impuestos indirectos, que son por naturaleza regresivos; llevan a cabo políticas para desestimular el vicio, que representa tantos problemas sociales, sobre todo a nivel de la juventud.

\section{Estructura de los impuestos municipales}

Los municipios del país recaudan 23 impuestos diferentes, de los cuales 3 de ellos concentran el $90 \%$ del total del recaudo, a saber: industria y comercio, predial y 
sobretasa a la gasolina. E1 10 \% restante corresponde al recaudo de otros impuestos, entre los que se destacan: alumbrado público, avisos y tableros, estampillas, degüello de ganado menor, sobretasa ambiental y delineación y urbanismo.

A continuación se hace un análisis de la caracterización de los principales impuestos municipales:

\title{
3.1. Impuesto de industria y comercio
}

El sustento normativo del impuesto de industria y comercio se encuentra definido en la Ley 14 de 1983, que en sus capítulos II y III reglamentan el tema, estableciendo el hecho imponible, la base gravable y la unificación de tarifas. Al respecto, la Ley estipula:

\begin{abstract}
El impuesto de industria y comercio recaerá, en cuanto a materia imponible, sobre todas las actividades comerciales, industriales y de servicio que ejerzan o realicen en las respectivas jurisdicciones municipales, directa o indirectamente, por personas naturales, jurídicas o por sociedades de hecho, ya sea que se cumplan en forma permanente u ocasional, en inmuebles determinados, con establecimientos de comercio o sin ellos (artículo 32).
\end{abstract}

Sobre la base gravable definida en el artículo anterior se aplicará la tarifa que determinen los concejos municipales dentro de los siguientes límites: del dos al siete por mil mensual para actividades industriales, y del dos al diez por mil mensual para actividades comerciales y de servicios.

Para el caso de Bogotá D.C., las normas aplicables son el decreto Ley 1421 de 1993 por el cual se dicta el régimen especial del Distrito Capital y el decreto 352 de 2002. Las normas referidas establecen:

- Que el periodo de causación sea bimestral.

- Que sobre la base gravable se aplique una tarifa única del 2 al 30 por mil.

- Que la base gravable sea conformada por los ingresos netos del contribuyente obtenidos durante el periodo anterior.

Este marco normativo especial para la ciudad de Bogotá explica el hecho de que algunas empresas que se encontraban radicadas en la capital se estén desplazando a municipios cercanos a ella. 
Con posterioridad a la Ley 14 de 1983, no se han presentado modificaciones importantes en materia de este impuesto, por lo que se puede señalar que el marco normativo del impuesto de industria y comercio está consagrado en dicha ley.

El segundo recaudo tributario más importante a nivel municipal es el impuesto predial, cuyo sustento normativo se encuentra definido así:

Constitución Política, artículo 317 estipula que el predial es el único impuesto que puede tener como base gravable el avalúo catastral.

Ley 14 de 1983, introdujo modificaciones en el manejo de los impuestos regionales y locales, modificando el rango de tarifas entre el 1 y el 16 por mil, dejando la posibilidad de gravar con una tarifa de hasta el 33 por mil los lotes urbanizables no urbanizados.

Decreto 3496 de 1983 reglamentario de la Ley 14 de 1983, introduce innovaciones como la creación de un nuevo procedimiento catastral denominado actualización.

Ley 75 de 1986, introduce algunos ajustes a los criterios sobre los avalúos contemplados en la Ley 14 de 1983.

Ley 44 de 1990 crea el Impuesto Predial Unificado, definiendo como base gravable el avalúo catastral o autoavalúo.

Ley 242 de 1995, revisó los reajustes anuales de los avalúos e introdujo el criterio de meta de inflación total.

Decreto Ley 1421 de 1993, señaló que a partir del año 1994 la base del impuesto sería el autoavalúo.

Ley 388 de 1997, proporciona las pautas de ordenamiento territorial y señala normas, procedimientos, parámetros y criterios para la elaboración de avalúos comerciales, además de que clasifica el suelo municipal en urbano, rural y de expansión urbana.

La sobretasa a la gasolina, se sitúa como el tercer impuesto municipal en orden de importancia y su sustento normativo se encuentra definido en:

Ley 105 de 1993, por la cual se autoriza a los municipios y a los distritos, para establecer una sobretasa máxima del $20 \%$ al precio del combustible automotor, con destino exclusivo a un fondo de mantenimiento y construcción de vías públicas y a financiar la construcción de proyectos de transporte masivo.

Ley 488 de 1998, que autoriza a los municipios a adoptar la sobretasa al consumo de gasolina motor extra y corriente, estableciendo la base del impuesto como el 
valor de referencia de venta al público por galón que certifique mensualmente el Ministerio de Minas y Energía.

Decreto 2653 del 29 de diciembre de 1998, estipula la obligatoriedad del Ministerio de Minas y Energía de expedir la certificación del valor de referencia por galón que regirá para cada uno de dichos productos en el siguiente periodo gravable.

Ley 681 de 2001, se reglamenta el régimen de concesiones de combustibles en las zonas de frontera. Posteriormente se aprueban otras normas complementarias respecto al tema, como son los decretos 1505 y 1900 de 2002, entre otros.

Una vez definido el sustento normativo de los principales impuestos municipales se establece su estructura, tomando como referencia el año 2013, que corresponde a la última vigencia que presenta cifras consolidadas de las finanzas públicas territoriales.

Para el año 2013 el recaudo tributario de todos los municipios del país llegó a la suma de $\$ 14,97$ billones (DNP, 2013), con el siguiente peso porcentual:

Figura 2. Participación porcentual impuestos municipales - 2013

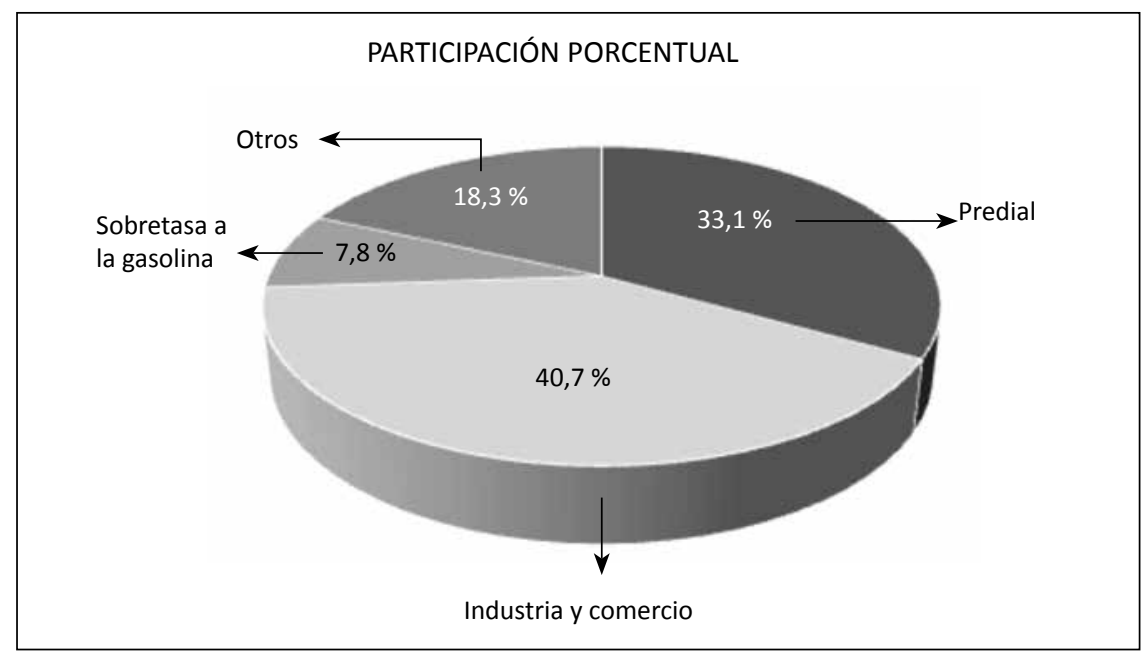

Fuente: desempeño fiscal 2013. DNP

De los anteriores impuestos el único que se clasifica como directo es el predial que representa el 33,1 \% del total recaudado, los otros impuestos se catalogan como indirectos y representan el restante $66,9 \%$, del total, lo que indica que la 
estructura tributaria municipal es altamente regresiva, lo que aumenta la inequidad en la redistribución de la riqueza en nuestro país.

Uno de los factores que influye notoriamente para que esta estructura sea regresiva, es la desactualización catastral que se presenta en la mayoría de municipios del país, sobre todo en predios que se clasifican como si fueran rurales siendo en la actualidad predios urbanos. El porcentaje de desactualización a nivel nacional es del 32,39\%, (IGAC, Subdirección de Catastro, Catastros Descentralizados) lo que no permite que se alcance una mayor dinámica en el recaudo del impuesto predial que cambie la regresividad de la estructura tributaria municipal.

A partir de estos resultados se infiere que la obligación de aportar a las cargas públicas territoriales, tanto a nivel departamental como municipal son inequitativas, por cuanto el sistema tributario territorial no se encuentra ajustado al principio constitucional de la progresividad, lo que demuestra que la desigualdad social del país no se soluciona por la función redistributiva de la hacienda pública.

\section{Conclusiones}

La estructura tributaria departamental presenta un profundo contrasentido, mientras que los gobiernos regionales buscan incrementar la capacidad para que la población incremente el consumo de bebidas alcohólicas, tabaco y otros, se llevan a cabo políticas para desestimular el vicio, que representa complejos problemas sociales, sobre todo para la juventud.

La estructura tributaria departamental es altamente regresiva, por cuanto sustenta sus recaudos en impuestos indirectos, favoreciendo la inequidad en la redistribución de la riqueza en nuestro país.

La estructura tributaria municipal, es igualmente regresiva, por cuanto sustenta sus recaudos en el impuesto de industria y comercio que es indirecto, lo que aumenta la inequidad en la redistribución de la riqueza en nuestro país.

La obligación de aportar a las cargas públicas territoriales, tanto a nivel departamental como municipal son inequitativas, por cuanto el sistema tributario territorial no se encuentra ajustado al principio constitucional de la progresividad, lo que determina que la desigualdad social del país no se soluciona por la función redistributiva de la hacienda pública.

La estructura de los impuestos territoriales en Colombia no contribuye a la obligación de aportar a las cargas públicas territoriales a través de la progresividad 
de los impuestos, por lo que no se están cumpliendo los preceptos teóricos de Smith y Musgrave, y mucho menos los preceptos constitucionales que tienen que ver con los deberes y obligaciones de los contribuyentes al financiamiento de los gastos e inversiones del Estado dentro de los principios de justicia y equidad, y tampoco con los principios tributarios de eficiencia y progresividad.

Se debe propender por una reforma tributaria territorial estructural, que corrija la regresividad en el recaudo de los diferentes impuestos tanto a nivel departamental como a nivel municipal, que conlleve a que la obligación de aportar a las cargas públicas territoriales sean equitativas.

\section{Referencias}

Constitución Política de Colombia 1991. (Edición 2014). Título XII, Del régimen económico y de la Hacienda pública.

Beltrán, G., y Torres, G. (2004). Finanzas Públicas. Bogotá, Colombia: Escuela Superior de Administración Pública-ESAP-.

Departamento Nacional de Planeación -DNP-. (2013). Desempeño fiscal de los departamentos y municipios vigencia 2012. Bogotá: Imprenta Nacional de Colombia.

Departamento Nacional de Planeación -DNP-. (2014). Desempeño fiscal de los departamentos y municipios vigencia 2013. Bogotá: Imprenta Nacional de Colombia.

Fino, G. (2012). Reforma tributaria municipal, una propuesta de crecimiento con equidad. Bogotá, Colombia: Editorial Temis.

IGAC- Subdirección de Catastro. (s.f.). Catastros Descentralizados.

Musgrave, R. (1959). The theory of public finance: a study in public economy. New York: McGraw-Hill.

Musgrave y Musgrave. (1992). Hacienda Pública Teórica y Aplicada. Editorial McGraw-Hill.

Ramírez, J. (2011). La Hacienda Pública en Colombia. Bogotá, Colombia: Librería Ediciones del Profesional Ltda.

Restrepo, J. C. (2008). Hacienda Pública. Bogotá: Universidad Externado, octava edición.

Silva, J., Páez, P., y Rodríguez, P. (2008). Finanzas Públicas Territoriales. Bogotá, Colombia: Escuela Superior de Administración Pública -ESAP-. 